\title{
Simple Test of Special Relativity Involving Fresnel Drag and Light Propagation in a Uniformly Moving Medium
}

\author{
Stephan J. G. Gift \\ Department of Electrical and Computer Engineering, The University of the West Indies, St Augustine, Trinidad and Tobago \\ Email: Stephan.Gift@sta.uwi.edu
}

How to cite this paper: Gift, S.J.G. (2021) Simple Test of Special Relativity Involving Fresnel Drag and Light Propagation in a Uniformly Moving Medium. Journal of Applied Mathematics and Physics, 9, 2660-2668. https://doi.org/10.4236/jamp.2021.911171

Received: October 6, 2021

Accepted: November 2, 2021

Published: November 5, 2021

Copyright $\odot 2021$ by author(s) and Scientific Research Publishing Inc. This work is licensed under the Creative Commons Attribution International License (CC BY 4.0).

http://creativecommons.org/licenses/by/4.0/

\begin{abstract}
In this paper, a simple test of special relativity involving light transmission through a uniformly moving medium and Fresnel's drag coefficient is discussed. It is shown using Fresnel drag that there is a difference in propagation time of two light beams travelling in opposite directions in such a medium and that this time difference is independent of the index of refraction of the medium. This result has been experimentally confirmed by Wang et al. but cannot be explained by special relativity. Fresnel drag in the context of ether theory provides a full and accurate explanation that is consistent with the existence of a preferred frame.
\end{abstract}

\section{Keywords}

Ether Theory, Special Relativity, Fresnel Drag, Sagnac Effect

\section{Introduction}

Special Relativity is today the accepted theory of space and time. Albert Einstein introduced this theory just over 100 years ago, following the failure of the Michelson-Morley experiment to detect movement through the luminiferous ether that was believed to be the medium of light transmission [1]. This experiment employed an interferometer that detected beams of light that travelled along orthogonal paths on a movable apparatus. The system was designed to determine the orbital speed of the Earth through the hypothesized ether, based on light speed changes arising from movement with or against the associated ether wind. On the basis of ether theory, the predicted fringe shift $\delta$ was found to be [2] 


$$
\delta=\frac{2 l}{\lambda} \frac{v^{2}}{c^{2}}
$$

where $v$ is the orbital speed of the Earth through the ether, $l$ is the arm length of the interferometer and $\lambda$ is the wavelength of the light. The observed fringe shift was significantly less than that expected. This essentially null result of this second-order $\left(\sim v^{2} / c^{2}\right)$ test ultimately led to the abandonment of the ether and the belief today that light needs no medium for its transmission. It should be noted that the Lorentz-Fitzgerald contraction hypothesis which is accepted as physically occurring in special relativity, does explain the null result of this experiment in the context of ether theory [2].

However in 1725, long before the Michelson-Morley experiment was conducted, Bradley discovered that starlight is shifted as a result of the orbital motion of the Earth. Consider the case of a star on the ecliptic pole corresponding to a direction that is $90^{\circ}$ to the Earth's orbital plane. The associated aberration arising from the orbital movement of the Earth when a normal telescope is aimed at the star is $\alpha$ as shown in Figure 1 [2] [3] [4]. This value, using classical velocity composition in the context of the luminiferous ether, is given by

$$
\alpha \simeq \tan \alpha=\frac{v}{c}
$$

where $v$ is the orbital speed of the Earth and $\alpha$ is in radians. This phenomenon, referred to as stellar aberration, along with knowledge of the Earth's orbital speed enabled Bradley to make one of the earliest measurements of the speed of light [2] [3] [4]. In fact, knowing light speed $c$ and aberration angle $\alpha$, this experiment can be used to detect the orbital movement of the Earth via Equation (2) just as Equation (1) was expected to do in the Michelson-Morley experiment. Therefore, the Bradley experiment effectively detects ether drift arising from the Earth's orbital motion, precisely the motion that the Michelson-Morley experiment failed to detect!

Arago in 1810 conducted a modified form of Bradley's experiment in an attempt to detect the movement of the Earth relative to the ether by a different mechanism [4]. It was based on the idea that the refraction of light would be influenced by the movement of the refractive substance. In this experiment, half of the objective lens of the telescope was covered with an achromatic prism so that

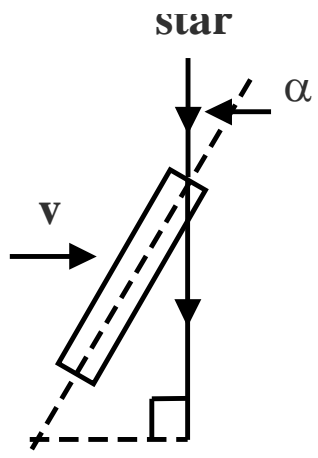

Figure 1. Stellar aberration discovered by Bradley. 
starlight could be collected either directly through the objective lens only or first through the prism. It was expected that the observation of these two images would involve different orientations of the telescope and that the difference between these two angles would be the deviation produced by the prism. The experiment yielded a null result as the passage of starlight through the prism resulted in no discernable effect. The movement of the Earth relative to the ether was therefore not detected using this approach involving refraction through a prism.

In 1818, Fresnel proposed an explanation for the null result of Arago's experiment. It is based on the idea that a body moving relative to the ether communicates a fraction $f$ of its movement to the light travelling within it. This constituted a partial drag of the light by the moving medium thereby adding to its velocity. This drag causes a change in the refraction of the light that exactly compensates for the change in aberration that was expected by passage through the prism [4]. The result is that the direction of the light emanating from the moving prism is the same as that without the prism and hence the null result of the experiment. This coefficient $f$ is referred to as Fresnel's drag coefficient.

In 1871 Airy conducted another version of Bradley's experiment again involving refraction but where the telescope was filled with water. The expected change in the position of the star was not observed and the null result was again explained by Fresnel's drag coefficient. The existence of Fresnel dragging resulting from movement of the medium through the ether was directly demonstrated by Fizeau in 1851 in an experiment using light transmission through moving water that produced the predicted phase shift rather than a null result. Hoek in 1868 conducted a modified form of Fizeau's experiment where the water was at rest relative to the laboratory. The result was a null result that was also fully explainable by Fresnel drag.

It is an interesting side note that despite the success of Bradley's experiment in effectively detecting ether drift associated with the orbital motion of the Earth using Equation (2), the failure (because of Fresnel drag) of the Arago, Airy and Hoek experiments to produce predicted effects has resulted in the erroneous view today that first-order $(\sim V / c)$ experiments such as Bradley's cannot reveal ether drift [5].

In this paper, we show that in ether theory the speed of light in a moving medium whether measured by an observer in the laboratory or one co-moving with the medium is not constant as required in special relativity. We use these results to derive the experimental finding by Wang et al. [6] that there is a difference in propagation time of two light beams travelling in opposite directions in such a medium as measured by a co-moving observer and this difference is independent of the index of refraction of the medium. We then show that special relativity is unable to produce this experimentally confirmed result and therefore the existence of a preferred frame is supported by the evidence.

\section{Fresnel's Drag Coefficient}

In order to determine Fresnel's Drag Coefficient, Airy's experiment which is a 
modified version of Bradley's experiment involving refraction where the telescope was filled with water can be used. Consider again the case of a star on the ecliptic pole corresponding to a direction that is $90^{\circ}$ to the Earth's orbital plane. The associated aberration arising from the orbital movement of the Earth when a normal telescope is aimed at the star is $\alpha$ as shown in Figure 2(a). For a telescope filled with water of refractive index $n$, the light entering the telescope undergoes refraction and travels down the water-filled telescope at a speed $c / n$ as shown in Figure 2(b). From Snell's law,

$$
n=\frac{\sin \beta}{\sin \delta} \simeq \frac{\beta}{\delta}
$$

where $\beta$ is the angle of incidence of the light on the telescope (corresponding to the aberration) and $\delta$ is the angle of refraction as the light enters the water within the telescope. Similar to Equation (2), in order that the image of the star be seen in the telescope, the angle of the telescope must be such that

$$
\delta \simeq \frac{v}{c / n}
$$

where $v$ is again the orbital velocity of the Earth. From (3) and (4),

$$
\beta=n \delta=n^{2} \frac{v}{c}
$$

Using (2) for $\alpha$, it follows that

$$
\beta-\alpha \simeq\left(n^{2}-1\right) \frac{v}{c}
$$

This means that a change in the position of the star between a normal telescope and the water-filled telescope is expected and this would enable the determination of the orbital velocity of the Earth. However, the experiment yielded a null result which means that the angle $\beta$ is equal to the angle $\alpha$ without the water. From (5) this results in

$$
\delta=\frac{\beta}{n}=\frac{\alpha}{n}
$$

In order to account for this using Fresnel dragging, as the Earth moves through the ether, the light travels through the water in the telescope at speed $c / n$ such that the time $t$ to travel the length $l$ of the telescope is given by

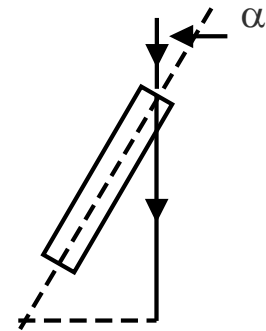

(a)

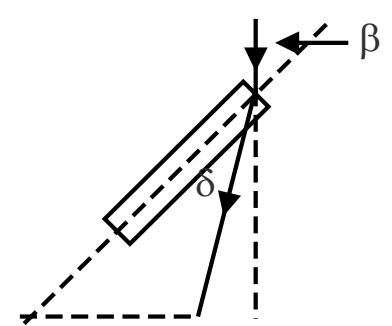

(b)

Figure 2. Airy's experiment using (a) a normal telescope; (b) a water-filled telescope. 


$$
t=\frac{l}{c / n}=\frac{n l}{c}
$$

In this time the telescope moves a distance $v t$ and hence the light displacement in the direction of motion must be equal to this telescope movement. The light refraction causes a displacement $l \tan \delta \simeq l \delta$ and the water in the telescope drags the light in the direction of motion resulting in a further displacement fvt. Hence

$$
v t=l \delta+f v t
$$

Using (8) for 1 and (7) for $\delta$ in (9) give

$$
v t=\frac{c t}{n} \frac{v}{n c}+f v t
$$

This yields

$$
f=1-\frac{1}{n^{2}}
$$

This is Fresnel's drag coefficient that has been directly confirmed using the Fizeau experiment [2] [3] [4].

\section{Light in a Moving Medium: Ether Theory}

Thus, for light travelling in a medium moving at velocity $V$ with respect to the laboratory (ether) in the same direction as the light as shown in Figure 3, then from Fresnel's Drag theory the light is dragged along by the moving medium giving the speed of light measured by an observer stationary in the laboratory as

$$
v_{\text {lab }}=\frac{c}{n}+f v=\frac{c}{n}+\left(1-\frac{1}{n^{2}}\right) v
$$

For light travelling in a direction opposite to the direction of movement of the medium the resulting light speed measured by the laboratory observer is given by

$$
v_{l a b}=\frac{c}{n}-f v=\frac{c}{n}-\left(1-\frac{1}{n^{2}}\right) v
$$

These values in (12) and (13) fully account for the positive fringe shift observed in the Fizeau experiment [2] [3] [4]. In the case where the observer is co-moving with the medium, if the medium is moving in the same direction as the light, then by velocity composition the speed of the light relative to the observer is

$$
v_{\text {medium }}=\frac{c}{n}+f v-v=\frac{c}{n}+\left(1-\frac{1}{n^{2}}\right) v-v=\frac{c}{n}-\frac{v}{n^{2}}
$$

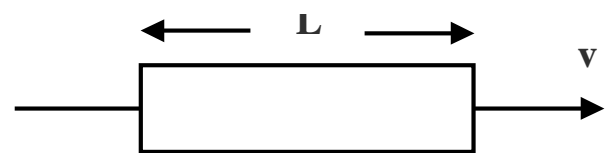

Figure 3. Medium Moving at velocity $v$. 
If the medium is moving in a direction opposite to that of the light, then by velocity composition the speed of the light relative to the co-moving observer is

$$
v_{\text {medium }}=\frac{c}{n}-f v+v=\frac{c}{n}-\left(1-\frac{1}{n^{2}}\right) v+v=\frac{c}{n}+\frac{v}{n^{2}}
$$

The velocities in (14) and (15) completely account for the null result in the Hoek experiment [2] [3] [4].

It is clear therefore that in the context of ether theory, the speed of light in a moving medium is not constant relative to a co-moving observer. Moreover, for light propagating in opposite directions in the moving medium of length $L$ as shown in Figure 3, the difference in transmission time as measured by the co-moving observer is given by

$$
\Delta t=\frac{L}{\frac{c}{n}-\frac{v}{n^{2}}}-\frac{L}{\frac{c}{n}+\frac{v}{n^{2}}} \simeq \frac{2 L v}{c^{2}}
$$

The result in (16) is that there is a transmission time difference between the two counter-propagating beams which is the Sagnac Effect in a moving medium. Additionally, this difference is independent of the index of refraction of the medium. This result has been experimentally confirmed by Wang et al. using a fiber optic interferometer and co-moving source and detector [6].

\section{Light in a Moving Medium: Special Relativity}

In 1907 von Laue showed that the Fresnel Drag coefficient is contained in special relativity as a consequence of the relativistic formula for the composition of velocities given by [4] [7]

$$
u=\frac{u^{\prime}+v}{1+\frac{u^{\prime} v}{c^{2}}}
$$

where $u^{\prime}$ is the velocity in the "moving" frame and $u$ is the velocity in the "stationary" frame. For the case under consideration in this paper, the light within the moving medium has a velocity $c / n$ relative to the medium which is moving at a velocity $v$ relative to the observer stationary in the laboratory. Therefore using (17), the velocity of the light measured by an observer stationary in the laboratory is given by

$$
v_{l a b}=\frac{\frac{c}{n}+v}{1+\frac{\frac{c}{n} v}{c^{2}}} \simeq \frac{c}{n}+\left(1-\frac{1}{n^{2}}\right) v
$$

for light travelling in the same direction as the medium and

$$
v_{l a b}=\frac{\frac{c}{n}-v}{1-\frac{\frac{c}{n} v}{c^{2}}} \simeq \frac{c}{n}-\left(1-\frac{1}{n^{2}}\right) v
$$


for light travelling in a direction opposite to the medium [3]. Equations (18) and (19) of special relativity are exactly Equations (12) and (13) of ether theory that explained Fizeau's experiment. In special relativity, these equations are derived without directly invoking Fresnel drag as in ether theory but the two results are identical to first-order. In the case where the observer is co-moving with the medium, the light within the moving medium has a velocity $c / n$ relative to the medium but the medium has zero velocity relative to the observer who is co-moving. Therefore, the velocity composition formula (17) gives the speed of the light relative to the co-moving observer as

$$
v_{\text {medium }}=\frac{\frac{c}{n} \pm 0}{1 \pm \frac{\frac{c}{n} \cdot 0}{c^{2}}}=\frac{c}{n}
$$

In the context of special relativity Equation (20) accounts for the null result of the Hoek experiment ([3]: p 83) since there is no motion of the medium relative to the laboratory, even though it is different from the corresponding result (14) and (15) in ether theory. Using (20), for light propagating in opposite directions in a moving medium of length $L$ as shown in Figure 3, the difference in propagation time as measured by the co-moving observer is given by

$$
\Delta t=\frac{L}{\frac{c}{n}}-\frac{L}{\frac{C}{n}}=0
$$

The result in (21) indicates no difference in transmission time for counter-propagating beams measured by a co-moving observer i.e. no Sagnac effect. This prediction by special relativity is contradicted by the Wang experiment where the Sagnac effect for counter-propagating light beams with co-moving light source and detector has been observed [6]. Therefore, special relativity makes an incorrect prediction and hence cannot be correct.

Ashby [8] employed the Lorentz transformations in an attempt to derive the result (16) within the framework of special relativity. He did so by comparing the propagation time of the signal measured by the co-moving observer with the propagation time measured in the underlying ECI frame. This approach is however flawed for two reasons. The first is that Ashby treats the observer as moving when in fact there is no movement of the observer relative to the medium in which the light is travelling. The second and more serious issue is Ashby's derivation determines light propagation time difference in the underlying ECI frame in which the medium is moving and not within the medium it where (16) applies and was experimentally confirmed. This derivation by Ashby involving analysis in the ECI frame seems to be an ad hoc one that was introduced by him in his 2004 publication [8] in order to produce the experimentally observed result (16) using special relativity. To the best of this author's knowledge, this procedure does not appear in the literature before the results of the experiment were published in 2003 [6] and in any event, is not valid. 


\section{Conclusion}

In this paper, ether theory has been used to account for the Sagnac effect in a moving medium for counter-propagating light beams, a phenomenon that has been experimentally confirmed [6]. Special Relativity on the other hand predicts a null result for counter-propagating beams in a moving medium and therefore cannot be a correct physical theory. We believe that the correct space-time theory is based on the Selleri transformations which involve a preferred frame [9] [10] [11]. Future research should therefore focus on incorporating Fresnel drag in the framework of these transformations. The prediction accuracy by ether theory involving Fresnel drag along with the failure of special relativity supports the existence of a preferred frame close to the Earth as claimed by Gift [12]. Such a frame corresponds to the well-known ECI frame of the Global Positioning System [12] [13].

\section{Conflicts of Interest}

The author declares no conflicts of interest regarding the publication of this paper.

\section{References}

[1] Michelson, A.A. and Morley, E.W. (1887) The relative Motion of the Earth and the Luminiferous Ether. American Journal of Science, S3-34, 333-345.

https://doi.org/10.2475/ajs.s3-34.203.333

[2] French, A.P. (1968) Special Relativity. Nelson, London.

[3] Robertson, H.P. and Noonan, T.W. (1968) Relativity and Cosmology. W.B. Saunders Company, Philadelphia.

[4] Ferraro, R. (2007) Einstein's Space-Time: An Introduction to Special and General Relativity. Springer-Verlag, New York.

[5] Stachel, J. (2005) 1905 and All That. Nature, 433, 215-217. https://doi.org/10.1038/433215a

[6] Wang, R., et al. (2003) Modified Sagnac Experiment for Measuring Travel-Time Difference between Counter-Propagating Light Beams in a Uniformly Moving Fiber. Physics Letters A, 312, 7-10. https://doi.org/10.1016/S0375-9601(03)00575-9

[7] Rindler, W. (2006) Relativity Special, General and Cosmological. 2nd Edition, Oxford University Press, New York.

[8] Ashby, N. (2004) The Sagnac Effect in the Global Positioning System. In: Rizzi, G. and Ruggiero, M.L., Eds., Relativity in Rotating Frames, Kluwer Academic Publishers, Dordrecht, The Netherlands. https://doi.org/10.1007/978-94-017-0528-8_3

[9] Selleri, F. (1996) Noninvariant One-way Velocity of Light. Foundations of Physics, 26, 641-664. https://doi.org/10.1007/BF02058237

[10] Selleri, F. (1997) Noninvariant One-way Velocity of Light and Locally Equivalent Reference Frames. Foundations of Physics Letters, 10, 73-83. https://doi.org/10.1007/BF02764121

[11] Gift, S.J.G. (2015) The Selleri Transformations and One-way Light Speed Measurement. Physics Essays, 28, 474-481. https://doi.org/10.4006/0836-1398-28.4.474

[12] Gift, S.J.G. (2021) Detection of the Ether Using the Global Positioning System. Ap- 
plied Physics Research, 13, 12-32. https://doi.org/10.5539/apr.v13n1p12

[13] Ashby, N. (2003) Relativity in the Global Positioning System. Living Reviews in Relativity, 6, Article No. 1. https://doi.org/10.12942/lrr-2003-1 